\title{
Burkholderia Measurement
}

National Cancer Institute

\section{Source}

National Cancer Institute. Burkholderia Measurement. NCI Thesaurus. Code C139113.

The determination of the amount of Burkholderia genus microorg anisms present in a sample. 